\title{
Association of Cardiac Galectin-3 Expression, Myocarditis, and Fibrosis in Chronic Chagas Disease Cardiomyopathy
}

Bruno Solano de Freitas Souza, ${ }^{* \dagger}$ Daniela Nascimento Silva,${ }^{\dagger}$ Rejane Hughes Carvalho, ${ }^{\dagger}$ Gabriela Louise de Almeida Sampaio, Bruno Diaz Paredes, ${ }^{\dagger}$ Luciana Aragão França, ${ }^{\dagger}$ Carine Machado Azevedo, ${ }^{* \dagger}$ Juliana Fraga Vasconcelos, ${ }^{* \dagger}$ Cassio Santana Meira, ${ }^{\star \dagger}$ Paulo Chenaud Neto, ${ }^{\dagger}$ Simone Garcia Macambira, ${ }^{* \dagger}$ Kátia Nunes da Silva, ${ }^{\dagger}$ Kyan James Allahdadi, Fabio Tavora, ${ }^{\S}$ João David de Souza Neto, ${ }^{\S}$ Ricardo Ribeiro dos Santos, ${ }^{\dagger}$ and Milena Botelho Pereira Soares ${ }^{* \dagger}$

From the Gonçalo Moniz Research Center, ${ }^{*}$ Oswaldo Cruz Foundation (FIOCRUZ), Salvador; the Center for Biotechnology and Cell Therapy, ${ }^{\dagger}$ São Rafael Hospital, Salvador; the Health Sciences Institute, ${ }^{\ddagger}$ Federal University of Bahia, Salvador; and Messejana Heart and Lung Hospital, ${ }^{\S}$ Fortaleza, Brazil

Accepted for publication January 19, 2017.

Address correspondence to Milena Botelho Pereira Soares, Ph.D., Centro de Pesquisas Gonçalo Moniz, Fundação Oswaldo Cruz, Rua Waldemar Falcão, 121, Candeal, Salvador, Bahia, Brazil CEP: 40296710. E-mail: milena@bahia. fiocruz.br.
Chronic Chagas disease cardiomyopathy, caused by Trypanosoma cruzi infection, is a major cause of heart failure in Latin America. Galectin-3 (Gal-3) has been linked to cardiac remodeling and poor prognosis in heart failure of different etiologies. Herein, we investigated the involvement of Gal-3 in the disease pathogenesis and its role as a target for disease intervention. Gal-3 expression in mouse hearts was evaluated during $T$. cruzi infection by confocal microscopy and flow cytometry analysis, showing a high expression in macrophages, $\mathrm{T}$ cells, and fibroblasts. In vitro studies using Gal-3 knockdown in cardiac fibroblasts demonstrated that Gal-3 regulates cell survival, proliferation, and type I collagen synthesis. In vivo blockade of Gal-3 with N-acetyl-D-lactosamine in $T$. cruzi-infected mice led to a significant reduction of cardiac fibrosis and inflammation in the heart. Moreover, a modulation in the expression of proinflammatory genes in the heart was observed. Finally, histological analysis in human heart samples obtained from subjects with Chagas disease who underwent heart transplantation showed the expression of Gal-3 in areas of inflammation, similar to the mouse model. Our results indicate that Gal-3 plays a role in the pathogenesis of experimental chronic Chagas disease, favoring inflammation and fibrogenesis. Moreover, by demonstrating Gal-3 expression in human hearts, our finding reinforces that this protein could be a novel target for drug development for Chagas cardiomyopathy. (Am J Pathol 2017, 187: 1134-1146; http://dx.doi.org/10.1016/j.ajpath.2017.01.016)
Chronic Chagas disease cardiomyopathy (CCC), caused by Trypanosoma cruzi infection, is an important cause of morbidity and mortality in endemic countries. It is estimated that approximately 7 million people are infected worldwide, with high prevalence in Latin America and growing incidence in developed countries because of globalization. ${ }^{1,2}$ It is estimated that the cardiac form of the disease occurs in approximately $20 \%$ to $30 \%$ of infected subjects. ${ }^{2}$ Antiparasitic drugs are effective during acute infection, but fail to improve established CCC. ${ }^{3,4}$ Besides standard heart failure treatment, patients with advanced CCC rely on heart transplantation, which is limited because of organ availability and complications relative to parasite reactivation after immunosuppression therapy. ${ }^{5}$
During CCC, cardiomyocytes are lost as a result of damage caused by immune responses directed to the parasites that persist in the heart, as well as to autoreactive cells directed to heart antigens. ${ }^{6,7}$ Although the mechanisms of pathogenesis are not completely understood, several studies indicate the involvement of type 1 helper T-cell lymphocytes associated with high production of interferon- $\gamma$ (IFN- $\gamma$ ), resembling a delayed hypersensitivity reaction. ${ }^{6}$ An association between progression to severe chronic forms and a high production of IFN- $\gamma$ was observed in

Supported by National Council for Scientific and Technological Development and Bahia Research Foundation.

Disclosures: None declared. 
patients with Chagas disease. ${ }^{8}$ Macrophages, a major cell population found in the inflammatory sites, can be activated by IFN- $\gamma$ and tumor necrosis factor- $\alpha$, two inflammatory cytokines overexpressed in the hearts of mice chronically infected with $T$. cruzi. Furthermore, several genes related to the inflammatory response are up-regulated in heart tissue during the chronic phase of $T$. cruzi infection. ${ }^{9}$

Previous studies suggested that activated macrophages secrete galectin-3 (Gal-3), a molecule involved in the pathogenesis of cardiac dysfunction. ${ }^{10}$ Gal-3 is a soluble $\beta$-galactoside binding lectin involved in a variety of cellular processes, including proliferation, migration, and apoptosis. ${ }^{11}$ The importance of this protein in the regulation of cardiac fibrosis and remodeling has been highlighted by the demonstration of its contribution to the development and progression of heart failure in different experimental settings. ${ }^{12-14}$ Serum Gal-3 concentrations are also increased in patients with acute decompensated heart failure. On the basis of these findings, the value of Gal-3 as a prognostic biomarker in patients with chronic heart failure has been investigated. ${ }^{15}$

Previously, we performed transcriptomic analysis in the cardiac tissue of mice chronically infected with $T$. cruzi, and found that Lgals3, the gene encoding for Gal-3, is among the most overexpressed genes. ${ }^{16}$ By immunofluorescence analysis, we showed that Gal-3 is mainly expressed in inflammatory cells in the hearts of $T$. cruzi-infected mice. We hypothesized that Gal-3 plays a role in the pathogenesis of CCC, contributing to the progression of inflammation and fibrosis. In the present study, we evaluated the expression of Gal-3 during T. cruzi infection in mice. Gal-3 expression was also investigated in human heart samples, to validate the expression of this protein in the human disease setting. Finally, we conducted in vitro and in vivo studies involving genetic and pharmacological blockades of Gal-3 to investigate its potential role in disease pathogenesis and its usefulness as a target for therapeutic development.

\section{Materials and Methods}

\section{Animal Procedures}

Six- to eight-week-old female C57BL/6 mice were used for T. cruzi infection and as normal controls. Galectin-3 C57BL/6 mice were used in cell adhesion experiments. All animals were raised and maintained at the animal facility of the Center for Biotechnology and Cell Therapy, Hospital São Rafael, in rooms with controlled temperature $\left(22^{\circ} \mathrm{C} \pm 2{ }^{\circ} \mathrm{C}\right)$ and humidity $(55 \% \pm 10 \%)$, and continuous air flow. Animals were housed in a 12-hour light/12-hour dark cycle (6АM to 6PM) and provided with standard rodent diet and water ad libitum. Animals were handled according to the NIH guidelines for animal experimentation. ${ }^{17}$ All procedures described had prior approval from the local institutional animal ethics committee at Hospital São Rafael (01/13).

\section{Trypanosoma cruzi Infection}

Trypomastigotes of the myotropic Colombian T. cruzi strain were obtained from culture supernatants of infected LLCMK2 cells, as previously described. ${ }^{9}$ Infection of C57BL/6 mice was performed by i.p. injection of $1000 \mathrm{~T}$. cruzi trypomastigotes in saline, and was confirmed through evaluation of parasitemia at different time points after infection.

\section{Pharmacological Blockade of Gal-3 with N-Lac}

C57Bl/6 female mice $(n=11)$ chronically infected with T. cruzi [6 months postinfection (m.p.i.)] were treated with N-acetyl-D-lactosamine (N-Lac) (Sigma-Aldrich, St. Louis, MO), $5 \mathrm{mg} / \mathrm{kg}$ per day, i.p. injections $3 \times$ per week, for 60 days. Chronically infected mice injected with saline $(n=10)$ and same age naïve mice $(n=8)$ served as controls. Functional analyses were performed, as described below. Mice were euthanized, by cervical dislocation under anesthesia with 5\% ketamine (König, São Paulo, Brazil) and 2\% xylazine (König Lab), the week after the final N-Lac injection. Heart samples were collected for real-time quantitative PCR and histological analysis. In another experiment, sucrose (Sigma-Aldrich) was administered in the same regimen as N-Lac to C57BL/6 mice, after 6 months of infection with T. cruzi. Heart samples were collected for histological analysis.

\section{Functional Analysis}

Electrocardiography was performed using the Bio Amp PowerLab System (PowerLab 2/20; ADInstruments, Sydney, Australia), recording the bipolar lead I. All animals were anesthetized by i.p. injection of $10 \mathrm{mg} / \mathrm{kg}$ xylazine and $100 \mathrm{mg} / \mathrm{kg}$ ketamine to obtain the records. All data were acquired for computer analysis using Chart 5 for Windows (ADInstruments). The electrocardiographic analysis included heart rate, PR interval, P wave duration, QT interval, QTc, and arrhythmias. The QTc was calculated as the ratio of QT interval by square roots of RR interval.

A motor-driven treadmill chamber for one animal (LE 8700; Panlab, Barcelona, Spain) was used to exercise the animals. The speed of the treadmill and the intensity of the shock (mA) were controlled by a potentiometer (LE 8700 treadmill control; Panlab). After an adaptation period in the treadmill chamber, the mice exercised at five different velocities $(7.2,14.4,21.6,28.8$, and $36.0 \mathrm{~m} /$ minute), with increasing velocity after 5 minutes of exercise at a given speed. Velocity was increased until the animal could no longer sustain a given speed and remained $>5$ seconds on an electrified stainless-steel grid. Total running distance was recorded.

\section{Morphometric Analysis}

Two months after the therapy, mice were euthanized as mentioned before and hearts were collected and fixed in $10 \%$ buffered formalin. Heart sections were analyzed by 
light microscopy after paraffin embedding, followed by standard hematoxylin and eosin staining. Inflammatory cells infiltrating heart tissue were counted using a digital morphometric evaluation system. Images were digitized using the slide scanner ScanScope (Leica, Wetzlar, Germany). Morphometric analyses were performed using Image Pro Plus software version 7.0 (Media Cybernetics, Rockville, MD). The inflammatory cells were counted in 10 fields $(\times 400$ magnification) per heart sample. The percentage of fibrosis was determined using Sirius red-stained heart sections and the Image Pro Plus version 7.0. Two blinded investigators performed the analyses (J.F.V. and C.M.A.).

\section{Immunofluorescence Analysis}

Frozen $(10 \mu \mathrm{m}$ thick) or formalin-fixed, paraffin-embedded ( $3 \mu \mathrm{m}$ thick) heart sections were obtained. Paraffin-embedded tissues were deparaffinized and submitted to a heat-induced antigen retrieval step by incubation in citrate buffer $(\mathrm{pH}=6.0)$. Then, sections were incubated overnight at $4^{\circ} \mathrm{C}$ with the following primary antibodies: anti-Gal-3, diluted 1:400 (Santa Cruz Biotechnology, Dallas, TX) and antiCD11b, diluted 1:400 (BD Biosciences, San Jose, CA). Next, the sections were incubated for 1 hour with secondary antibodies anti-goat IgG Alexa Fluor 488-conjugated and anti-rat IgG Alexa Fluor 594-conjugated (1:400; ThermoFisher Scientific, Waltham, MA). Immunostaining for in vitro experiments was performed in cardiac fibroblasts or bone marrow-derived macrophages plated on coverslips. The cells were fixed with paraformaldehyde $4 \%$ and incubated with the primary antibodies: goat anti-Gal-3, diluted 1:400 (Santa Cruz Biotechnology), or rabbit anti-collagen type I, diluted 1:50 (Novotec, Lyon, France). On the following day, sections were incubated for 1 hour with phalloidin conjugated with Alexa Fluor 633 or 488 conjugated, diluted 1:50, mixed with the secondary antibodies anti-goat IgG Alexa Fluor 488conjugated (1:400) or anti-rabbit IgG Alexa Fluor 568conjugated (1:200; all from ThermoFisher Scientific), respectively. Nuclei were stained with DAPI (VectaShield mounting medium with DAPI H-1200; Vector Laboratories, Burlingame, CA). The presence of fluorescent cells was determined by observation on a FluoView 1000 confocal microscope (Olympus, Tokyo, Japan) and A1+ confocal microscope (Nikon, Tokyo, Japan). Quantifications of Gal- $3^{+}$cells were performed in 10 random fields captured under $\times 400$ magnification, using the Image Pro Plus software version 7.0.

\section{Flow Cytometry Analysis}

Control and T. cruzi-infected mice were euthanized, hearts were collected, perfused with phosphate-buffered saline (PBS) to remove blood cells, and processed by enzymatic digestion using $0.1 \%$ collagenase IV (Sigma-Aldrich) and $10 \mu \mathrm{g} / \mathrm{mL}$ DNase (Roche, Basel, Switzerland), for 40 minutes, at $37^{\circ} \mathrm{C}$. To evaluate the subpopulations of digested cardiac tissue samples, cell suspensions were allowed to pass through a $70-\mu \mathrm{m}$ cell strainer (BD Biosciences) and counted. Aliquots of $10^{6}$ cells were used for each test tube and $1 \mu \mathrm{L}$ of Fc blocking reagent (BD Biosciences) was added. The fluorochromeconjugated antibody panels used for each subpopulation were: i) T lymphocytes: CD45-APC-Cy7, CD3-APC, CD4PE-Cy5, CD8-PE (BD Biosciences); ii) macrophages: CD45-APC-Cy7, CD11b-APC (eBiociences, San Diego, CA); iii) fibroblast/fibrocyte: CD45-APC-Cy7, vimentin-APC (Cell Signaling, Danvers, MA). Each antibody was diluted as suggested on the product data sheet. Samples were incubated for 20 minutes at room temperature in the dark. For intracellular staining of Gal-3, samples were washed once in PBS and CytoFix/CytoPerm kit (BD Biosciences) were used as directed on data sheet protocol. Anti-Gal-3-PE (R\&D Systems, Minneapolis, MN) antibody was added to macrophages and fibroblast/fibrocyte sample tubes, whereas nonconjugated anti-Gal-3 (Santa Cruz Biotechnology) was added on T lymphocyte sample tube and its detection was performed by addition of anti-mouse IgG-Alexa Fluor 488 (ThermoFisher Scientific). Each incubation step was performed during 30 minutes at room temperature in the dark. Samples were washed twice and resuspended in PBS and added with Hoecsht 33258 to exclude cell debris from analysis. Apoptosis was evaluated by annexin V-PI assay. Cells were harvested from culture flasks by adding TrypLE solution (ThermoFisher Scientific) and incubating for 5 minutes at $37^{\circ} \mathrm{C}$. Cell suspensions were collected and washed with PBS by centrifugation at $300 \times g$. After discarding supernatant, pellets were resuspended in binding buffer (ThermoFisher Scientific) and cells were counted. Apoptosis assays were performed using annexin-V-APC and PI (BD Biosciences) according to the manufacturer's recommendations. Sample acquisition was performed using a BD LSRFortessa SORP cytometer (BD Biosciences) using BD FacsDiva software version 6.2 (BD Biosciences). Ten thousand events were acquired per sample, and the data were analyzed using FlowJo software version 7.5 (FlowJo Enterprise, Ashland, OR).

\section{Real-Time RT-PCR}

Total RNA was isolated from heart samples with TRIzol reagent (ThermoFisher Scientific) and the concentration was determined by spectrophotometry. High Capacity cDNA Reverse Transcription Kit (ThermoFisher Scientific) was used to synthesize cDNA of $1 \mu \mathrm{g}$ RNA following manufacturer's recommendations. Real-time RT-PCR assays were performed to detect the expression levels of Tbet (Mm_00450960_m1), Gata3 (Mm_00484683_m1), Tnf (Mm_00443258_m1), Ifng (Mm_00801778_m1), Illo (Mm_00439616_m1), Foxp3 (Mm_00475162_m1), Lgals3 (Mm_00802901_m1), and MMP9 (Mm_00444299_m1). Other primer sequences used in real-time PCR analyses: Collal: 5'-GTCCCTCGACTCCTACATCTTCTGA$3^{\prime}$ (forward) and 5'-AAACCCGAGGTATGCTTGATCTGTA' (reverse); Ccnd1: 5'-TCCGCAAGCATGCACAGA-3' 
(forward) and $5^{\prime}$-GGTGGGTTGGAAATGAACTTCA-3' (reverse); Cavl: 5'-GGCACTCATCTGGGGCATTTA- ${ }^{\prime}$ (forward) and 5'-CTCTTGATGCACGGTACAACC- ${ }^{\prime}$ (reverse). The real-time RT-PCR amplification mixtures contained a $20 \eta$ g template cDNA, TaqMan Master Mix (10 $\mu \mathrm{L}$ ) and probes, constituting a final volume of $20 \mu \mathrm{L}$ (all from ThermoFisher Scientific). All reactions were run in duplicate on an ABI7500 Sequence Detection System (ThermoFisher Scientific) under standard thermal cycling conditions. The mean $\mathrm{Ct}$ values from duplicate measurements were used to calculate expression of the target gene, while normalized to an internal control (Gapdh) using the 2-DCt formula. Experiments with $\mathrm{CVs}>5 \%$ were excluded. A nontemplate control and nonreverse transcription controls were also included.

\section{Design of shRNAs and Production of Lentiviral Vectors}

To stably knock down Lgals3 expression, we designed shRNA against different regions of the Lgals3 coding sequence, and a scramble shRNA as control. Target sequences were designed using the online tool siRNA Wizard software version 3.1 (Invivogen, San Diego, CA). All suggested sequences were blasted against the mouse RNA reference sequence database, and the three with the lowest degree of homology to other sequences were selected: Lgals3_shRNA1 5'-GATTTCAGGAGAGGGAATGAT-3'; Lgals3_shRNA2 5'-GGTCAACGATGCTCACCTACT-3'; Lgals3_shRNA3 5'-CATGCTGATCACAATCATGG-3'; and one Lgals3_scrbl_shRNA 5'-AGGTATGAGTCGAGATTGAGA-3'. Sense and antisense single strands, containing the target sequence, a loop sequence (TCAAGAG), and restriction enzyme sites for Mlu at the sense sequence and $\mathrm{ClaI}$ at the antisense sequence, were synthesized separately. The annealing of both strands to form double-stranded shRNAs was performed by incubating $2.5 \mu \mathrm{mol} / \mathrm{L}$ from the sense and antisense strand of each shRNA in $10 \mathrm{mmol} / \mathrm{L}$ Tris$\mathrm{HCl}(\mathrm{pH} 7.5), 0.1 \mathrm{~mol} / \mathrm{L} \mathrm{NaCl}$, and $1 \mathrm{mmol} / \mathrm{L}$ EDTA at $95^{\circ} \mathrm{C}$ for 5 minutes and then allowing the reaction to cool down to room temperature for at least 2 hours. The double-stranded shRNAs were then phosphorylated using T4 PNK (New England Biolabs, Ipswich, MA) following manufacturer protocol. The shRNAs were cloned into the pLVTHM lentiviral vector (Addgene plasmid 12247), specifically designed for gene knockdown with shRNAs, ${ }^{18}$ after the vector was linearized by digestion with MluI and ClaI (New England Biolabs) according to the manufacturer instructions. Each of the produced shRNA constructs were confirmed by sequencing using ABI 3500 platform (ThermoFisher Scientific).

For lentiviral vector production, HEK293 FT cells were cotransfected with each of the shRNA constructs, plus psPAX2 (Addgene plasmid 12260) and pMD2.G (Addgene plasmid 12247) for production of the lentivirus particles, in a proportion of 3:2:1. Viral supernatants were harvested 48 and 72 hours later, pooled, centrifuged to remove cell debris, filtered through $0.45-\mu \mathrm{m}$ filters (Millipore, Billerica, MA), and concentrated by ultracentrifugation. Cardiac fibroblasts were transduced with the lentivirus by overnight incubation in medium containing lentiviral particles and $6 \mu \mathrm{g} / \mathrm{mL}$ polybrene. Knockdown efficiency for each shRNA was evaluated by real-time quantitative PCR using TaqMan probes for Lgals3 (mm00802901_m1), Gapdh (mm99999915_g1), Actb (mm00607939_s1), and Hprt (mm00496968_m1) and TaqMan Universal PCR master mix (ThermoFisher Scientific), according to the manufacturer's instructions. Assay was performed in triplicate, and the empty vector was used as control. Ct for Lgals3 was normalized taking into account the geometric mean of the $\mathrm{Ct}$ for Gapdh, Actb, and Hprt $(\Delta \mathrm{Ct})$. The relative expression was then calculated by the normalized $\mathrm{Ct}$ between each Lgals3 shRNA construct and the empty vector $(\Delta \Delta \mathrm{Ct})$.

\section{In Vitro Studies with Cardiac Fibroblasts and Bone Marrow-Derived Macrophages}

Cardiac fibroblasts were isolated from hearts of adult C57BL/6 mice, euthanized as described above. Hearts were minced into pieces of $1 \mathrm{~mm}$ and incubated with $0.1 \%$ collagenase type A (Sigma-Aldrich) at $37^{\circ} \mathrm{C}$ for 30 minutes, under constant stirring. The cell suspension was passed through a $70-\mu \mathrm{m}$ cell strainer (BD Biosciences), and plasticadherent cells were selected by 1 hour incubation in gelatincoated flasks (Sigma-Aldrich). Nonadherent cells from supernatant were removed and adherent cells were cultured with Dulbecco's modified Eagle's medium supplemented with $10 \%$ fetal bovine serum and 1\% penicillin and streptomycin (all from ThermoFisher Scientific), in a humidified incubator at $37^{\circ} \mathrm{C}$ with $5 \% \mathrm{CO}_{2}$. Culture medium was changed every 3 days, and cells were trypsinized (trypsinEDTA $0.05 \%$; ThermoFisher Scientific) when $80 \%$ confluence was reached. Cell cycle studies were performed with CFSE Cell Proliferation Kit (ThermoFisher Scientific), according to the manufacturer's instructions. Proliferation of cardiac fibroblasts was assessed by the measurement of ${ }^{3} \mathrm{H}$-thymidine uptake. Cells were plated in 96-well plates, at a density of $10^{4}$ cells/well, in a final volume of $200 \mu \mathrm{L}$, in triplicate, and cultured in the absence or presence of $30 \mu \mathrm{g} / \mathrm{mL}$ rmGal-3 (R\&D Systems), with or without 1\% modified citrus pectin (ecoNugenics, Santa Rosa, CA). After 24 hours, plates were pulsed with $1 \mu \mathrm{Ci}$ of methyl $-{ }^{3} \mathrm{H}$ thymidine (PerkinElmer, Waltham, MA) for 18 hours, and proliferation was assessed by measurement of ${ }^{3} \mathrm{H}$-thymidine uptake by using a Chameleon $\beta$-plate counter (Hydex, Turku, Finland). Proliferation capacity of Gal-3 knockdown and control cell lines was compared by ${ }^{3} \mathrm{H}$-thymidine incorporation, using the same procedures.

To obtain macrophages, bone marrow cells were harvested from femurs of C57BL/6 mice by flushing with cold RPMI 1640 medium. Bone marrow cells were induced to differentiate into macrophages by culture in RPMI 1640 supplemented with $10 \%$ fetal bovine serum (ThermoFisher Scientific), $50 \mathrm{U} / \mathrm{mL}$ of penicillin, $50 \mu \mathrm{g} / \mathrm{mL}$ of streptomycin, $2.0 \mathrm{~g} / \mathrm{L}$ of sodium bicarbonate, $25 \mathrm{mmol} / \mathrm{L}$ HEPES, $2 \mathrm{mmol} / \mathrm{L}$ 
glutamine, and 30\% supernatant obtained from X63-GM$\mathrm{CSF}^{19}$ cell line culture, at $37^{\circ} \mathrm{C}$ and $5 \% \mathrm{CO}_{2}$. Cells were cultured for 7 days, with half medium changes every 3 days. Differentiated macrophages were plated onto 24-well plates and incubated in medium alone or with $1 \mu \mathrm{g} / \mathrm{mL}$ lipopolysaccharide (Sigma-Aldrich) with or without $50 \mathrm{ng} / \mathrm{mL} \mathrm{IFN- \gamma}$ (R\&D Systems). After 24 hours, macrophages were detached using a cell scraper and analyzed for Gal-3 expression by flow cytometry, as described above.

\section{Human Samples}

The procedures involving human samples received prior approval by the local Ethics committee at Hospital São Rafael (approval number 51025115.3.0000.0048). Samples were obtained at Messejana Hospital in Fortaleza, Ceará, a medical center specialized for heart transplantation in Brazil. Fragments of explanted hearts from three patients with Chagas disease, confirmed by serological assay, were obtained from left ventricle and septum. Samples were processed in paraffin and stained with hematoxylin and eosin and Sirius Red, or used for immunostaining for detection of Gal-3, as described above.

\section{Lymphoproliferation Assay}

Splenocyte suspensions, obtained from C57B1/6 mice, were prepared in Dulbecco's modified Eagle's medium supplemented with $10 \%$ fetal bovine serum and $50 \mu \mathrm{g} / \mathrm{mL}$ of gentamicin. Splenocytes were cultured in 96-well plates at $1 \times 10^{6}$ cells/well, in triplicate, and lymphocyte proliferation was stimulated or not with concanavalin A $(2 \mu \mathrm{g} / \mathrm{mL}$; Sigma-Aldrich) or Dynabeads mouse T-activator CD3/ CD28 (ThermoFisher Scientific), according to the manufacturer's instructions. Cell proliferation was induced in the absence or presence of various concentrations of N-Lac $(10,1$, and $0.1 \mu \mathrm{mol} / \mathrm{L})$. After 48 hours of incubation, $1 \mu \mathrm{Ci}$ of ${ }^{3} \mathrm{H}$-thymidine was added to each well, and the plate was incubated for 18 hours. Plates were frozen at $-70^{\circ} \mathrm{C}$, then thawed and transferred to UniFilter-96 GF/B PEI coated plates (PerkinElmer) with the assistance of a cell harvester. After drying, $50 \mathrm{~mL}$ of scintillation cocktail was added in each well, sealed and plate read at liquid scintillation microplate counter. Dexamethasone (Sigma-Aldrich; $10 \mu \mathrm{mol} / \mathrm{L}$ ) was used as positive control. Three independent experiments were performed.

\section{En Face Leukocyte Adhesion Assay}

The aorta from the thoracic region and spleens were removed from wild-type and galectin-3 knockout C57BL/6 mice. Fragments of approximately $1 \mathrm{~mm}^{2}$ were placed with the intimal side up in 96-well plates previously coated with Matrigel (Corning Inc., Corning, NY) for 30 minutes at $37^{\circ} \mathrm{C}$. Endothelium was activated by incubation with $500 \mathrm{ng} / \mathrm{mL}$ lipopolysaccharide (Sigma), whereas the splenocyte suspension with $2 \mu \mathrm{g} / \mathrm{mL}$ concanavalin A (Sigma)

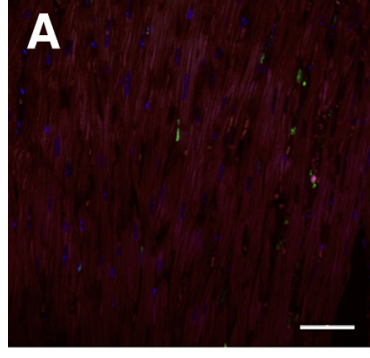

D
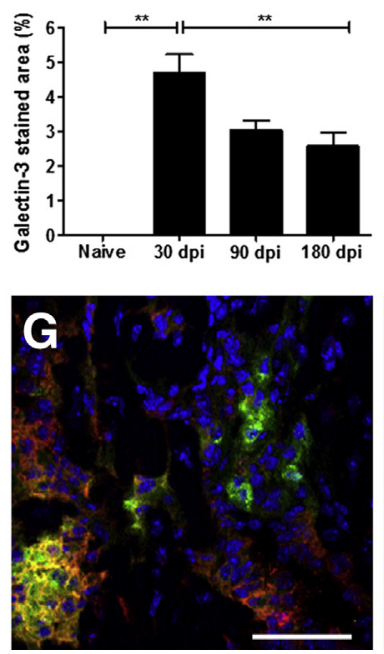

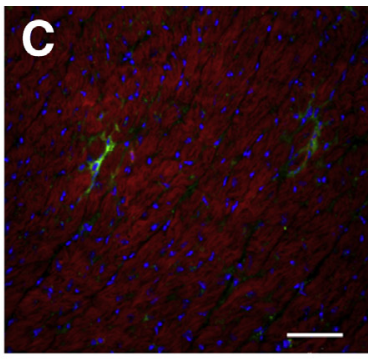

$\mathbf{F}$
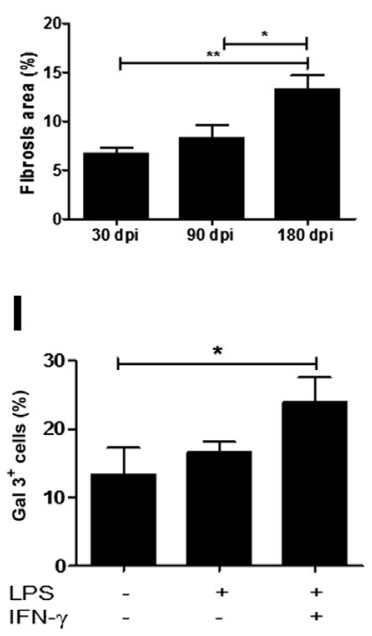

Figure 1 Gal-3 is overexpressed in mouse hearts after Trypanosoma cruzi infection. Confocal microscopy analysis demonstrated the presence of Gal- $3^{+}$cells (green), mainly in areas of inflammatory infiltrates, in naïve (A), at 1 (B) and 6 (C) months postinfection (m.p.i.). Cardiac muscle was stained for actin- $\mathrm{F}$ (red), and nuclei were stained with DAPI (blue). D: The cardiac expression of Gal-3 peaked at 1 m.p.i., but remained elevated during the chronic phase of infection, when compared to naïve mice. E: A similar pattern is observed for the number of inflammatory cells infiltrating the heart. F: However, the percentage of fibrosis increased with time. G: Most cells expressing Gal-3 (green) coexpressed the monocyte/macrophage marker CD11b (red). H: Cardiac fibroblasts isolated by enzymatic digestion of heart tissue also express Gal-3 (green). Actin- $\mathrm{F}$ is seen in red, and nucleus in blue. I: Bone marrowderived macrophages stimulated in vitro with proinflammatory (M1) inductors interferon- $\gamma$ $(\mathrm{IFN}-\gamma)$ and lipopolysaccharide (LPS) increase the expression of Gal-3. Data are expressed as means \pm SEM. ${ }^{*} P<0.05,{ }^{* *} P<0.01$, and ${ }^{* *} * P<0.001$. Scale bars $=50 \mu \mathrm{m}$. dpi, days postinfection. 


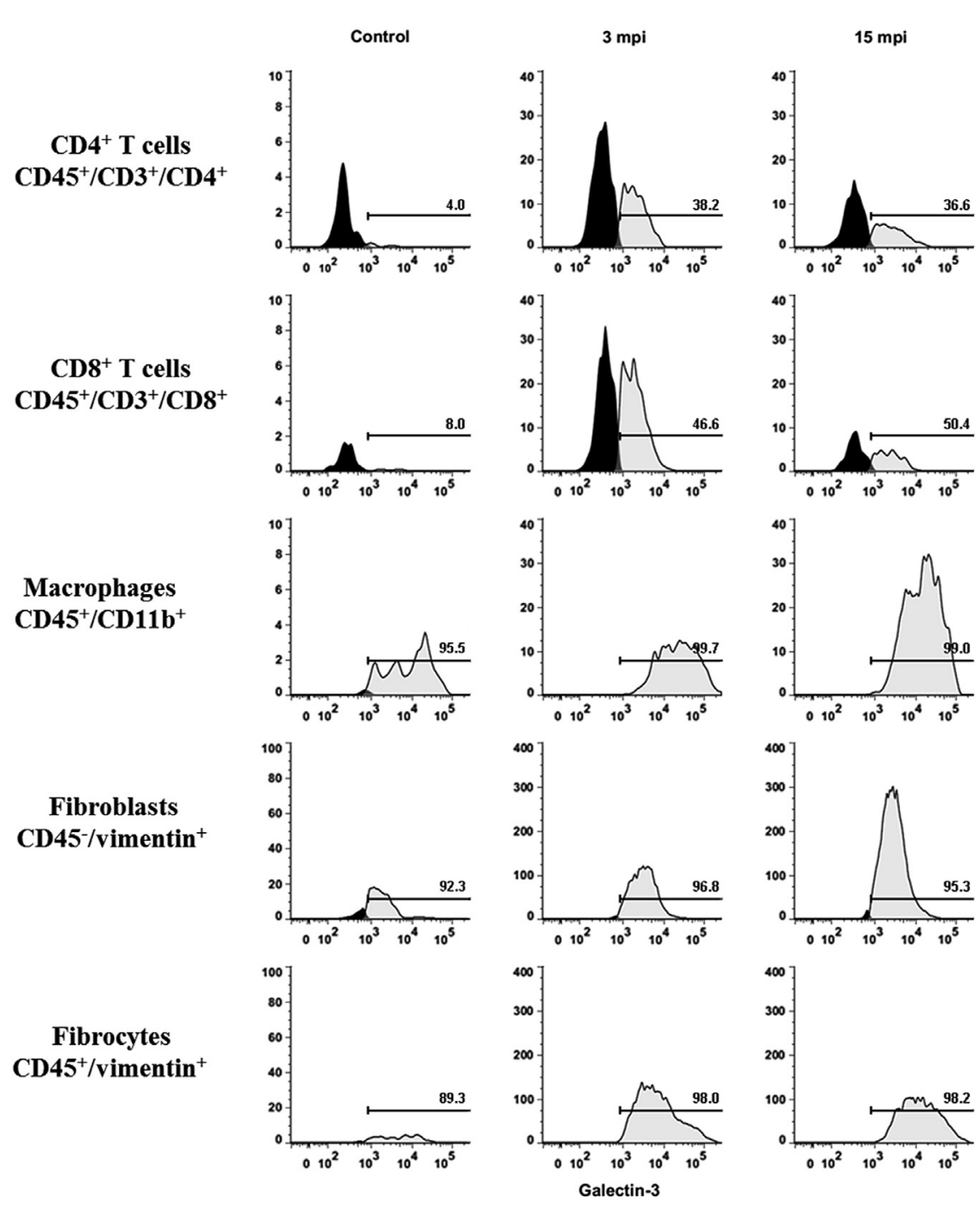

Figure 2 Gal-3 is increased in different cell types involved in inflammation and tissue repair. Histograms showing flow cytometry analysis from digested heart tissue, obtained from naïve and infected mice, at 3 and 15 months postinfection (m.p.i.). Gal- $3^{+} \mathrm{T} \mathrm{CD4}^{+}$and $\mathrm{CD}^{+}$lymphocytes expressing Gal-3 are increased at 3 and 15 m.p.i. when compared to naïve controls. Most macrophages $\left(\mathrm{CD} 45^{+} / \mathrm{CD}_{11} \mathrm{~b}^{+}\right)$, fibroblasts $\left(\mathrm{CD} 45^{-}\right)$ vimentin $\left.{ }^{+}\right)$, and bone marrow-derived fibrocytes $\left(\mathrm{CD}_{4} 5^{+}\right.$vimentin $\left.{ }^{+}\right)$express Gal-3 $(>90 \%)$ in all groups, but the mean fluorescence intensity increases with time of infection.

and $500 \mathrm{ng} / \mathrm{mL}$ lipopolysaccharide, during a period of 6 hours.

Activated splenocytes were incubated with $1 \mu \mathrm{mol} / \mathrm{L}$ Celltracker Fluorescent Probes (Life Technologies) in serum-free RPMI 1640 medium (Gibco) for 30 minutes and washed three times, before adhesion to the endothelium. Splenocytes $\left(5 \times 10^{5} /\right.$ well $)$ were plated and incubated with the aortic endothelium fragments for 30 minutes at $37^{\circ} \mathrm{C}$ in the presence or absence of $10 \mu \mathrm{mol} / \mathrm{L} \mathrm{N}$-Lac (SigmaAldrich). Plates were then carefully washed three times with warm Hanks' balanced salt solution to remove the nonadherent cells. Three replicates were used for each treatment. Different random areas per well were acquired using a digital camera from an inverted fluorescence microscope. Fluorescent cells were quantified using ImagePro.

\section{Inhibition of Cell Migration Assay}

C57BL/6 mice, 8 to 12 weeks old, were submitted to euthanasia by cervical dislocation under anesthesia. Spleens

were collected, minced, cells were resuspended in PBS and passed through a $70-\mu \mathrm{m}$ cell strainer. The cells were resuspended and maintained in RPMI 1640 medium (ThermoFisher Scientific), without serum, supplemented with $2 \mathrm{mmol} / \mathrm{L}$ L-glutamine (ThermoFisher Scientific), 0.1\% RMPI 1640 vitamin solution (Sigma Aldrich), $1 \mathrm{mmol} / \mathrm{L}$ sodium pyruvate, $10 \mathrm{mmol} / \mathrm{L}$ HEPES, $50 \mu \mathrm{mol} / \mathrm{L}$ 2-mercaptoetanol, and penicillin/streptomycin solution (all from ThermoFisher Scientific). Splenocytes were incubated in starvation during 24 hours at $37^{\circ} \mathrm{C}$ and $5 \% \mathrm{CO}_{2}$, in the presence or absence of $10 \mu \mathrm{mol} / \mathrm{L} \mathrm{N}$-Lac. Migration assay was performed using the QCM Chemotaxis Cell Migration Assay, 24-well 3- $\mu \mathrm{m}$ pore (Millipore), according to the manufacturer's instructions. Briefly, splenocytes were counted and $10^{7}$ cells in $250 \mu \mathrm{L}$ were placed in the upper chamber, in serum-free medium, in the presence or absence of N-Lac. RPMI 1640 medium supplemented with $10 \%$ fetal bovine serum (ThermoFisher Scientific) with or without $10 \mu \mathrm{mol} / \mathrm{L} \mathrm{N}$-Lac was placed in the bottom chamber. Cells present in the bottom chamber were counted after overnight incubation. 


\section{Statistical Analysis}

All continuous variables are presented as means \pm SEM. Continuous variables were tested for normal distribution using Kolmogorov-Smirnov test. Parametric data were analyzed using unpaired $t$ tests, for comparisons between two groups, and one-way analysis of variance, followed by Bonferroni post hoc test for multiple-comparison test, using Prism 6.0 (GraphPad, La Jolla, CA). $P<0.05$ was considered statistically significant.

\section{Results}

\section{Gal-3 Expression Is Increased during Experimental T. cruzi Infection}

We first analyzed the expression of Gal-3 in mouse heart sections obtained at different time points of infection. Trypanosoma cruzi infection led to increased expression of Gal- $3^{+}$cells in the myocardium compared to naïve controls, as shown by confocal microscopy (Figure 1, A-C). Quantification of Gal-3 expression showed a significant increase in all time points analyzed, in comparison with uninfected controls (Figure 1D). The number of Gal- $3^{+}$cells was higher at the peak of parasitemia (1 m.p.i.), when an intense acute inflammatory response is found in the heart (Figure 1E). The numbers of Gal- $3^{+}$cells during the chronic phase were sustained, whereas the percentage of fibrosis increased with time (Figure 1F). The population of Gal- $3^{+}$cells in the heart included macrophages $\left(\mathrm{CD}_{11} \mathrm{~b}^{+}\right.$cells) (Figure $\left.1 \mathrm{G}\right)$ and cardiac fibroblasts (Figure 1H). To investigate the role of proinflammatory signals in the expression of Gal-3 by macrophages, we performed in vitro studies to analyze the expression of Gal-3 in activated macrophages. Bone marrow-derived macrophages activated with IFN- $\gamma$ and Toll-like receptor 4 ligand lipopolysaccharide had an increased expression of Gal-3, as demonstrated by flow cytometry analysis (Figure 1I).

To better characterize the cell populations expressing Gal-3, we performed flow cytometry analysis of cells isolated from hearts of $T$. cruzi-infected mice (Figure 2). Both $\mathrm{CD}^{+}$and $\mathrm{CD}^{+} \mathrm{T}$ cells had increased Gal-3 expression at 3 and 15 m.p.i. when compared to uninfected controls. In addition, macrophages, characterized as $\mathrm{CD} 45^{+} / \mathrm{CD} 11 \mathrm{~b}^{+}$, composed the cell populations expressing the higher mean fluorescence intensity of Gal-3 (Figure 2). Gal-3 was expressed at low levels in fibroblasts (vimentin ${ }^{+} / \mathrm{CD} 45^{-}$) in control hearts, and was increased by $52.5 \%$ at 3 m.p.i. Gal-3 expression intensity in fibroblasts at 15 m.p.i. returned to levels similar to those found in controls. However, a significant increase in Gal-3 expression was detected in a population of vimentin ${ }^{+} / \mathrm{CD} 45^{+}$cells, characterized as bone marrow-derived fibrocytes, at 3 and 15 m.p.i., when compared to controls (Figure 2).

\section{Expression of Gal-3 in the Hearts of Subjects with CCC}

To evaluate if the presence of Gal- $3^{+}$cells in the myocardium of infected mice could be translatable to the human
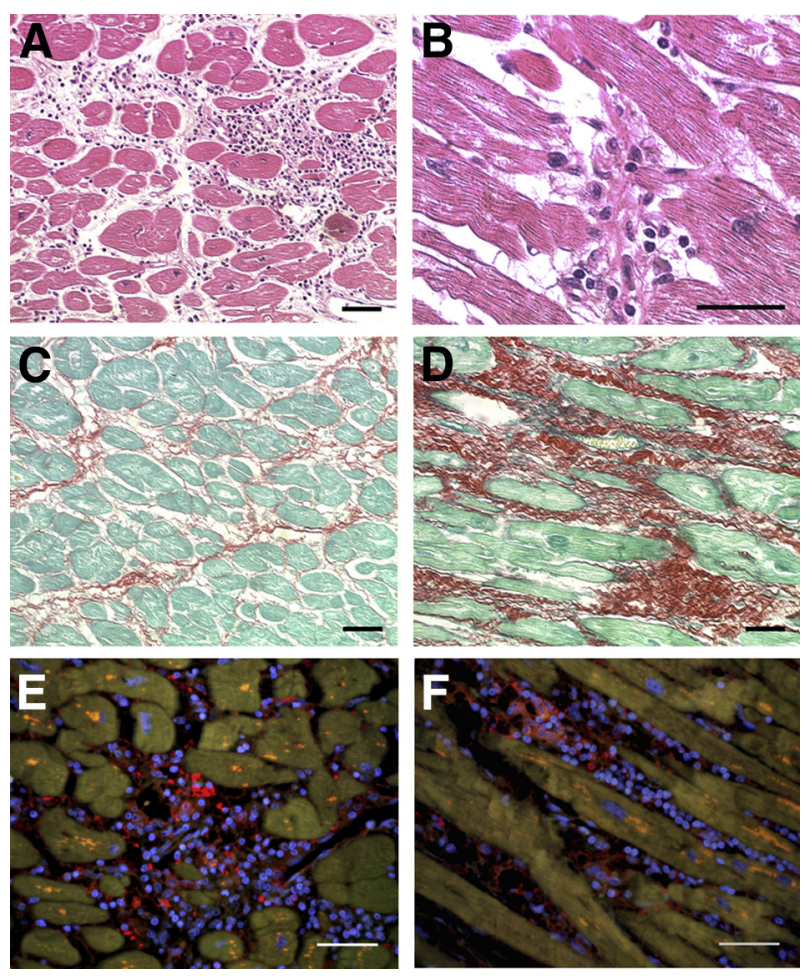

Figure 3 Gal-3 expression in heart samples from subjects with end-stage Chagas cardiomyopathy. Representative images obtained from explanted heart sections of two subjects with end-stage Chagas cardiomyopathy who underwent heart transplantation. Heart sections were stained with hematoxylin and eosin, showing inflammatory infiltrates composed of mononuclear cells surrounding myofibers (A) and in areas of myocytolysis (B). Heart sections stained with Sirius red showing areas of mild (C) and extensive (D) cardiac fibrosis. E and F: Confocal microscopy analysis from two different subjects, showing Gal- $3^{+}$cells (red) in areas of inflammatory infiltrates. Nuclei are stained with DAPI (blue). Scale bars $=50 \mu \mathrm{m}(\mathbf{A}-\mathbf{C}, \mathbf{E}$, and $\mathbf{F}) ; 25 \mu \mathrm{m}(\mathbf{D})$.

disease, we performed analysis in human heart samples obtained from explants of subjects with chronic Chagas disease cardiomyopathy who underwent heart transplantation. Heart sections were prepared and stained with hematoxylin and eosin for histological analysis, demonstrating the presence of foci of myocarditis, with an inflammatory infiltrate composed mainly of mononuclear cells, leading to the destruction of myofibers (Figure 3, A and B). In addition, an extensive area of diffuse fibrotic scar was found in Sirius red-stained sections (Figure 3, C and D). The expression of Gal-3 in human heart samples was evaluated by analysis using confocal microscopy. We observed the presence of cells, within the inflammatory foci and surrounding the myofibers, expressing variable levels of Gal-3 (Figure 3, E and F).

\section{Gal-3 Is a Major Regulator of Fibroblast Function}

On the basis of the findings of increased Gal-3 expression in fibroblasts during the development of CCC, we performed in vitro studies aiming at investigating the role of Gal-3 on different aspects of the biology of these cells. Cardiac 

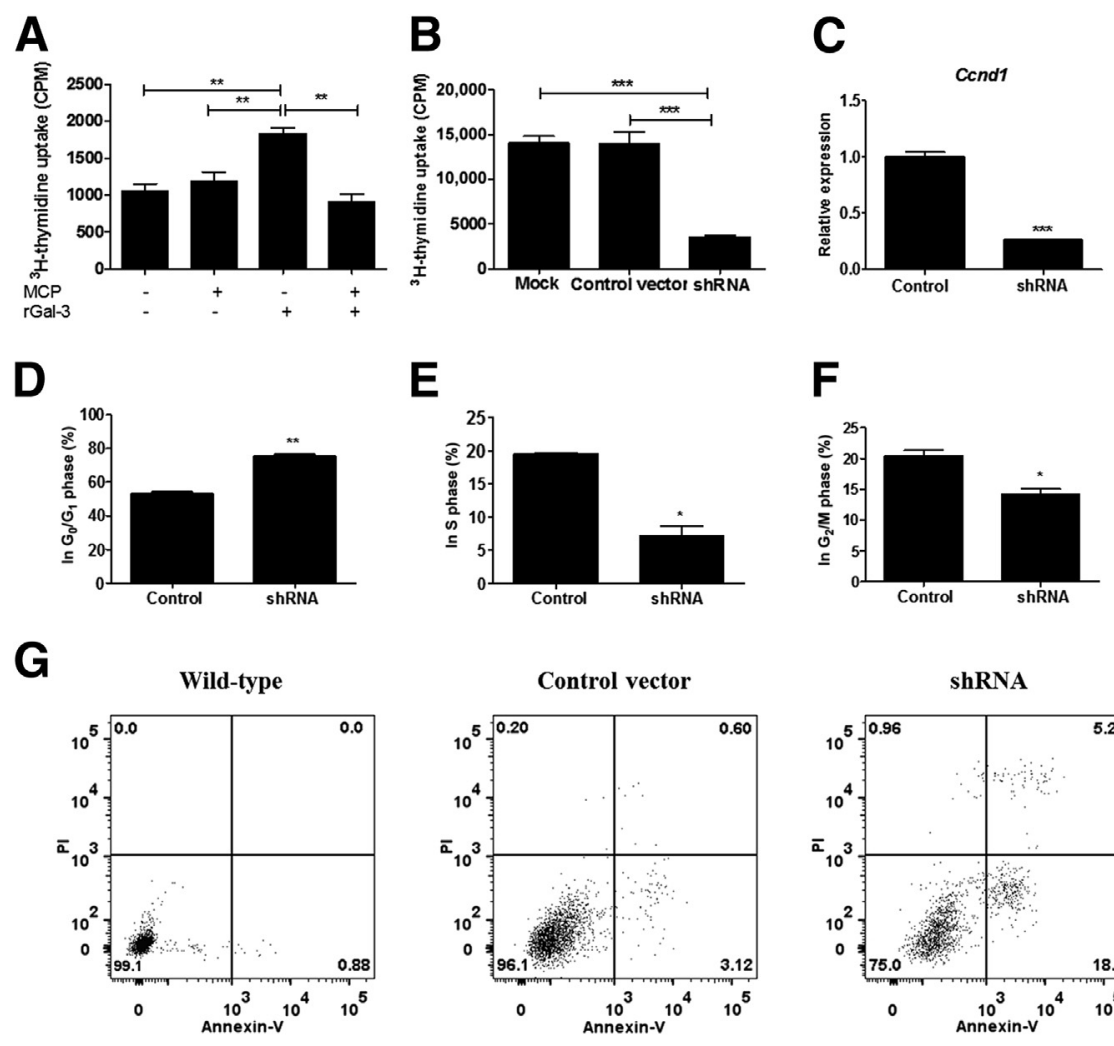

Figure 4 Gal-3 is crucial for cardiac fibroblast proliferation and survival. Recombinant Gal-3 was added to the cardiac fibroblast culture medium and cell proliferation was measured by ${ }^{3} \mathrm{H}$-thymidine incorporation assay. A: Extracellular Gal-3 induced cardiac fibroblast proliferation, which is abolished by addition of modified citrus pectin, a Gal-3 binding partner. B: Gal-3 knockdown in cardiac fibroblasts markedly reduces cell proliferation, as evaluated by ${ }^{3} \mathrm{H}$-thymidine incorporation assay. C: A reduction in gene expression of cyclin D1 is found by real-time quantitative PCR analysis. Cell cycle analysis was performed by flow cytometry with carboxyfluorescein succinimidyl ester assay, demonstrating that Gal-3 knockdown in cardiac fibroblasts is associated with cell cycle arrest in $\mathrm{G}_{0}$ / $G_{1}$ phases (D) and reduced number of cells in the $S$ (E) and $G_{2} / M(F)$ phases. G: Gal-3 knockdown is associated with increased frequency of apoptosis, as evaluated by annexin $V$ assay. Data are expressed as means \pm SEM. ${ }^{*} P<0.05$, ${ }^{* *} P<0.01$, and ${ }^{* * * P}<0.001$. CPM, counts per minute; PI, propidium iodide. fibroblasts isolated from mouse hearts were incubated with mouse recombinant Gal-3 to evaluate their proliferative rate. We found that exogenous recombinant Gal-3, at a micromolar concentration, increased the proliferation of cardiac fibroblasts, whereas addition of modified citrus pectin, a binding partner of Gal-3, blocked the effect of Gal-3 (Figure 4A).

Considering that this effect was observed at high concentrations of exogenous Gal-3, and given the high expression of intracellular Gal-3 in cardiac fibroblasts in experimental CCC, we evaluated the role of endogenous Gal-3 in cardiac fibroblasts. We generated lentiviral vectors encoding shRNA targeting the Lgals3, together with green fluorescence protein expression as a reporter gene. Then, cardiac fibroblasts were transduced by lentiviral infection, resulting in the knockdown of Gal-3. The efficiencies of lentiviral infection and knockdown were confirmed by green fluorescence protein reporter gene expression and by quantification of Gal-3 gene and protein expressions by realtime quantitative PCR and immunofluorescence analysis, respectively ( $>90 \%$ ) (Supplemental Figure S1, A-E). More important, Gal-3 knockdown in cardiac fibroblasts led to a down-regulation of type I collagen expression (Supplemental Figure S1, F-H).

Gal-3 knockdown was associated with a significant reduction in the proliferative rate of cardiac fibroblasts (Figure 4B). This finding was accompanied by a reduction of cyclin D1 gene expression (Figure 4C). Analysis of caveolin-1 gene expression did not show alterations when control or Gal-3 knockdown cells were compared (data not shown). Flow cytometry analysis showed cell cycle arrest in Gal-3 knockdown when compared to control cells (increased percentage of $\mathrm{G}_{0} / \mathrm{G}_{1}$ and decreased $\mathrm{S}$ and $\mathrm{G}_{2} / \mathrm{M}$ phases) (Figure 4, D-F).

To determine whether Gal-3 knockdown also affects cell survival, we evaluated the frequency of apoptosis in the culture of cardiac fibroblasts, by annexin V/PI staining and flow cytometry analysis. A higher percentage of apoptotic cells was detected in cultures of cardiac fibroblasts with Gal-3 knockdown when compared to controls (Figure 4G).

Treatment with the Gal-3 Blocking Agent N-Lac Reduces Inflammation and Fibrosis in Experimental CCC

To study the role of Gal-3 on the pathogenesis of CCC, we performed a pharmacological blockade of Gal-3 using $\mathrm{N}$-Lac (Figure 5A), during the chronic phase of the infection, when cardiac fibrosis is significantly increased (6 and 8 m.p.i.). We performed functional evaluations (electrocardiographic analysis and treadmill test) before treatment (6 m.p.i.) and after the treatment with N-Lac (8 m.p.i.). Trypanosoma cruzi infection caused the development of arrhythmias and cardiac conduction disturbances, such as atrioventricular block, ventricular tachycardia, and ventricular bigeminy. Treatment with $\mathrm{N}-\mathrm{Lac}$ did not alter the frequencies or the severity of arrhythmias when compared to those found in saline-treated controls (Table 1). Regarding 

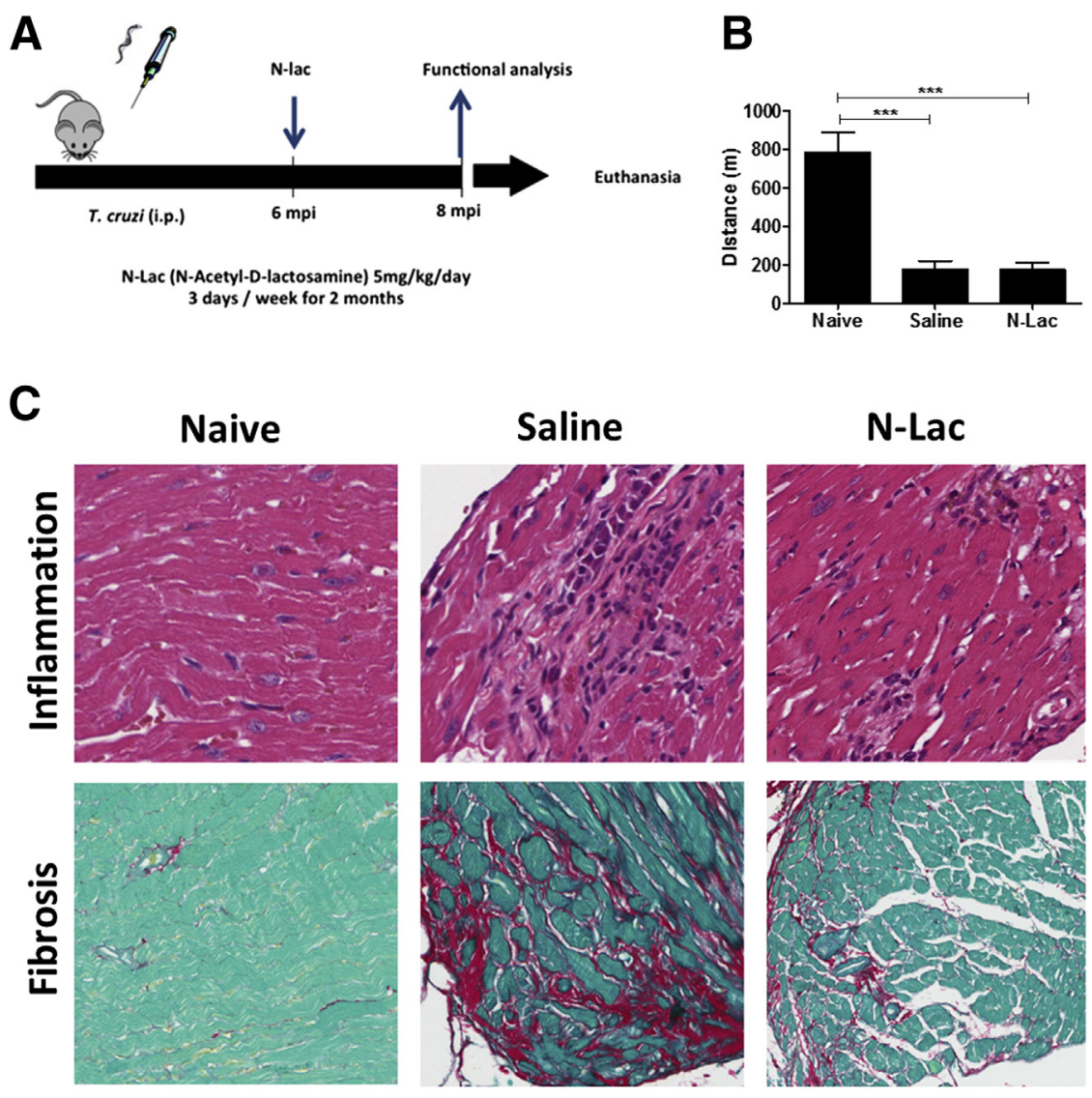

Figure 5 In vivo pharmacological blockade of Gal-3 during the chronic phase of experimental Trypanosoma cruzi infection reduces inflammation and fibrosis. A: Experimental design. B: A lack of functional recovery was observed by analysis of performance in treadmill test 2 months after the beginning of treatment with N-Lac. C: A significant reduction in the intensity of cardiac inflammation and fibrosis is observed in heart sections of mice treated with $\mathrm{N}$-Lac stained with hematoxylin and eosin (top row) and Sirius red (bottom row). Quantifications of the number of inflammatory cells infiltrating the heart (D), cardiac fibrosis area (E), showing histological improvement in N-Lac treated mice. Data are expressed as means \pm SEM. ${ }^{*} P<0.05,{ }^{*} p<0.01$, and ${ }^{*} * * P<0.001$. Original magnification, $\times 200$ (C). m.p.i., months postinfection.
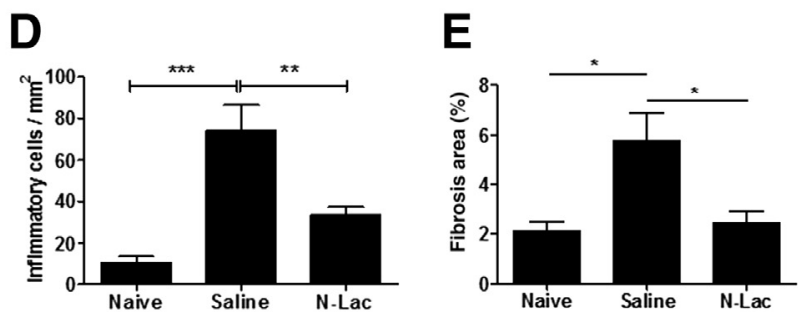

the exercise capacity, T. cruzi-infected mice had an impaired performance when compared to uninfected controls 6 months after infection (data not shown). N-Lac treatment did not cause any improvement in exercise capacity, because mice treated with this Gal-3 blocker had similar performance in treadmill test to saline-treated mice and a reduced capacity when compared to uninfected controls (Figure 5B).

Histological analysis demonstrated the presence of inflammatory infiltrate in the hearts of mice infected with $T$. cruzi, mainly composed of mononuclear cells. The number of inflammatory cells infiltrating the heart, however, was significantly reduced in N-Lac-treated mice, compared to saline-treated controls (Figure 5, C and D). In addition, the percentage of heart fibrosis was significantly reduced after $\mathrm{N}$-Lac treatment when compared to saline-treated mice (Figure 5, C and E). In addition, a control experiment was performed in which $T$. cruzi-infected mice were treated in the same regimen with sucrose. Morphometric analysis in the hearts of sucrose-treated mice did not show reduction of inflammatory cells and the fibrotic area in sucrose-treated mice when compared to those treated with saline (Supplemental Figure S2).

To investigate whether N-Lac caused modulation of inflammatory mediators, we performed gene expression analysis in the heart tissue (Figure 6). N-Lac-treated mice had reduced gene expression of the inflammatory cytokines tumor necrosis factor- $\alpha$ and IFN- $\gamma$ when compared to saline-treated mice. The regulatory cytokine IL-10 was increased in $T$. cruzi-infected mice when compared to uninfected controls, both in saline as well as in N-Lactreated mice. Moreover, the gene expression of transcription factors T-bet, GATA-3, and FoxP3, associated with T-cell subtypes type 1 helper $\mathrm{T}$ cell, type 2 helper $\mathrm{T}$ cell, and $\mathrm{T}$ regulatory cell, respectively, was increased by $T$. cruzi infection and reduced in mice treated with N-Lac. The gene 
Table 1 ECG Analysis in Uninfected and Trypanosoma cruziInfected Mice

\begin{tabular}{|c|c|c|c|c|}
\hline ECG findings & $\begin{array}{l}\text { Uninfected } \\
(n=7)\end{array}$ & $\begin{array}{l}\text { Pretreatment } \\
(n=19)\end{array}$ & $\begin{array}{l}\text { Saline } \\
(n=9)\end{array}$ & $\begin{array}{l}\text { N-Lac } \\
(n=10)\end{array}$ \\
\hline No alterations & $7 / 7$ & $1 / 19$ & & \\
\hline Atrial overload & & $1 / 19$ & $1 / 9$ & \\
\hline IACD & & & $2 / 9$ & \\
\hline $\mathrm{JR}$ & & $1 / 19$ & $1 / 9$ & \\
\hline AVB first degree & & $6 / 19$ & $3 / 9$ & $2 / 10$ \\
\hline AVB third degree & & $5 / 19$ & $2 / 9$ & $3 / 10$ \\
\hline SVT & & $2 / 19$ & $1 / 9$ & $1 / 10$ \\
\hline $\begin{array}{l}\text { Ventricular } \\
\text { bigeminy }\end{array}$ & & & & $3 / 10$ \\
\hline Isorhythmic AVD & & $2 / 19$ & $1 / 9$ & $1 / 10$ \\
\hline AVD & & & $1 / 9$ & \\
\hline IVCD & & $1 / 19$ & $1 / 9$ & \\
\hline
\end{tabular}

$A V B$, atrioventricular block; AVD, atrioventricular dissociation; ECG, electrocardiography; IACD, intra-atrial conduction delay; IVCD, intraventricular conduction delay; JR, junctional rhythm; N-Lac, N-acetyl-Dlactosamine; SVT, supraventricular tachycardia.

expression of chemokine ligand 8 (modified citrus pectin 2) and the chemokine receptor CCR5, which are increased by $T$. cruzi infection, was also reduced after N-Lac treatment. More important, treatment with N-Lac reduced the gene expression of Gal-3 in the hearts of T. cruzi-infected mice (Figure 6).

To better investigate the mechanisms by which N-Lac caused reduction of inflammation, we performed lymphoproliferation and migration assays. Mouse splenocytes were stimulated in vitro with concanavalin A or anti-CD3/CD28.
Addition of $\mathrm{N}-\mathrm{Lac}$ at the highest concentration tested $(10 \mu \mathrm{mol} / \mathrm{L})$ caused a small reduction of lymphoproliferation stimulated by both polyclonal activators (Figure 7, A and B). In contrast, the positive control dexamethasone inhibited the proliferation induced by both stimuli. Last, we tested the effects of N-Lac in adhesion of leukocytes to the endothelium and in cell migration. The adhesion of leukocytes to aorta endothelium in an en face assay was significantly blocked by N-Lac using cells and endothelium from wild-type mice, but not from galectin-3 knockout mice (Figure 7C). In fact, cell adhesion of galectin-3 knockout mice was similar to that of pharmacological blockade with N-Lac in wild-type cells (Figure 7C), In addition, the presence of N-Lac significantly inhibited leukocyte migration in a transwell system (Figure 7D).

\section{Discussion}

Gal-3 is a multifunctional lectin that can be found in various cells and tissues, and is detected in the nucleus, cytoplasm, as well as in the extracellular compartment. ${ }^{11}$ Notably, Gal-3 may have different, concordant, or opposite actions depending on the cell type and whether it is present in the extracellular or intracellular compartments. ${ }^{11}$ Previous studies from our group and others have shown a correlation between inflammation and fibrosis in the heart and Gal-3 expression. ${ }^{16,32,34}$ Moreover, host expression of Gal-3 is required for $T$. cruzi adhesion and invasion in human cells. ${ }^{20}$ In the present study, we demonstrated the expression of Gal3 in different cell populations and its role in the promotion
A
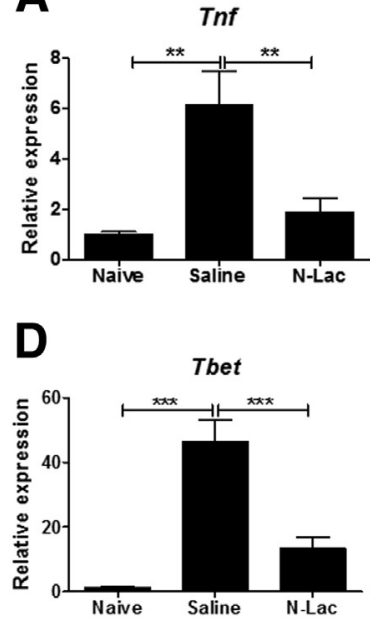

G

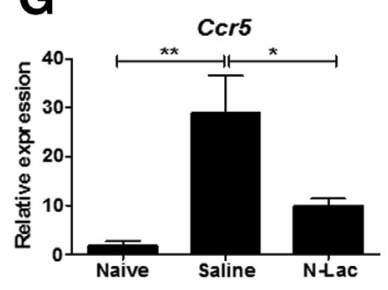

B

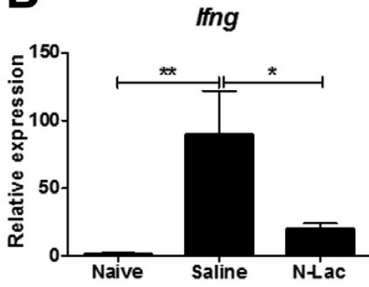

$E$

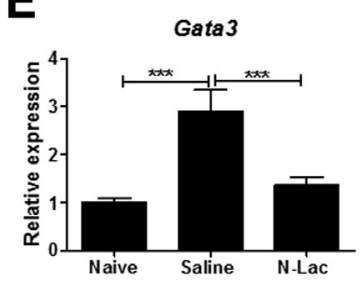

$\mathbf{H}$

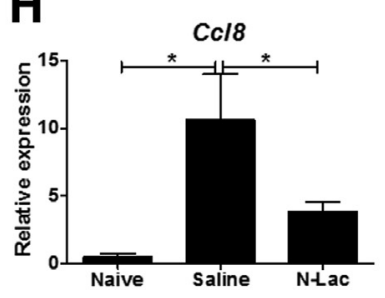

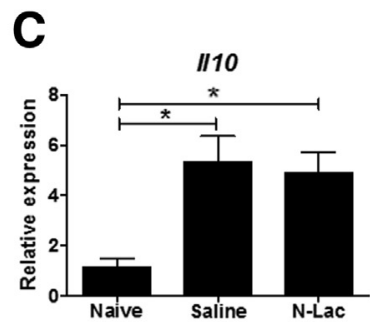

$F$
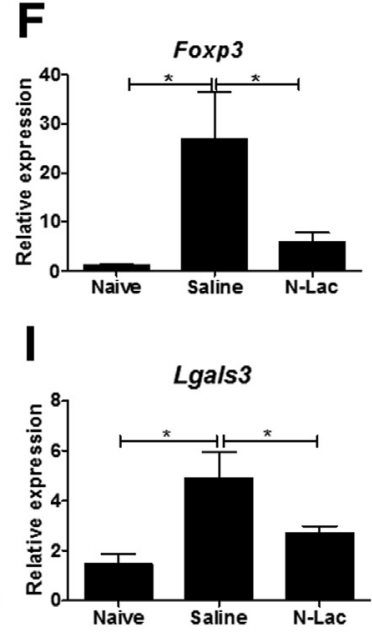

Figure 6 Modulation of gene expression in chagasic heart after $\mathrm{N}$-Lac treatment. real-time RT-PCR analysis of gene expression in the heart tissue demonstrates that N-Lac treatment is associated with a reduction of inflammatory cytokines tumor necrosis factor (TNF)- $\alpha$ (A) and interferon (IFN) $-\gamma(\mathbf{B})$, and does not alter the expression of IL-10 (C), when compared to salinetreated mice. T-lymphocyte subtype-specific transcription factors associated with type 1 helper T cell (T-bet; D), type 2 helper T cell (GATA-3; E), and $\mathrm{T}$ regulatory cell $(\mathrm{FOXP3} ; \mathrm{F})$ are reduced in $\mathrm{N}$-Lac-treated mice. The expression of genes associated with leukocyte migration and chemotaxis CCR5 (G), chemokine ligand $8(\mathbf{H})$, and Gal-3 (I) is also reduced after N-Lac treatment. Data are expressed as means \pm SEM. ${ }^{*} P<0.05$, ${ }^{* *} P<0.01$, and ${ }^{* * *} P<0.001$ 
A

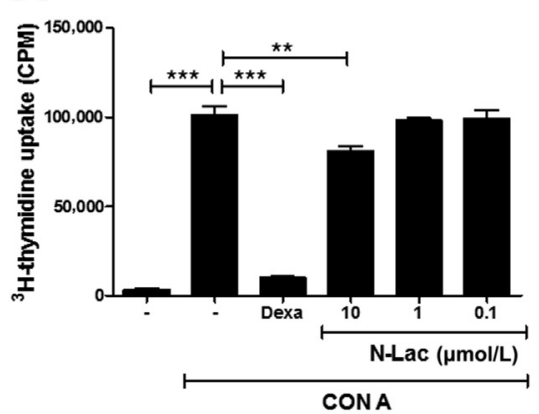

C

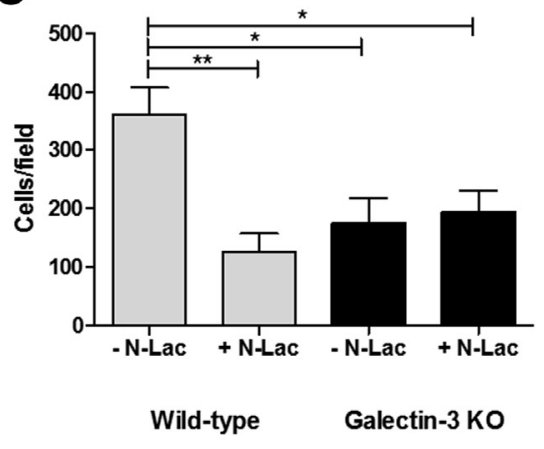

B

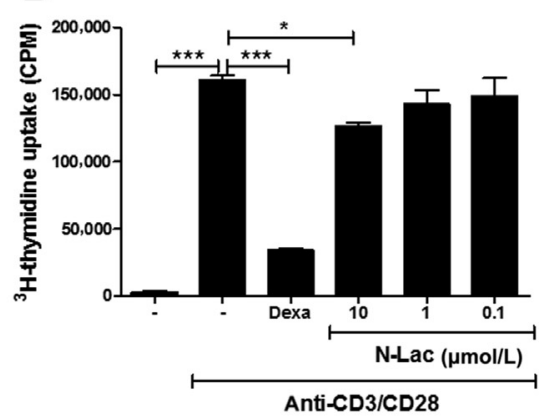

D

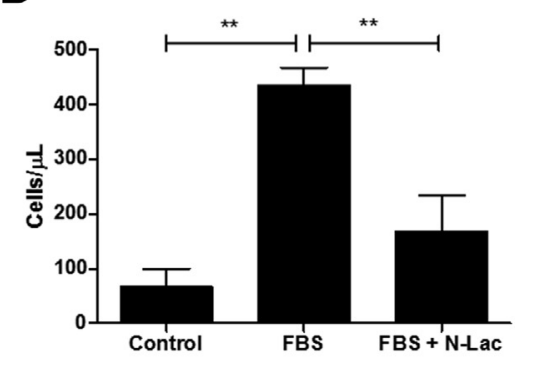

Figure 7 Effects of N-Lac on splenocyte proliferation and migration in vitro. Mouse splenocytes obtained from naïve $\mathrm{C} 57 \mathrm{BL} / 6$ mice were stimulated with concanavalin $\mathrm{A}(\operatorname{Con} \mathrm{A} ; \mathrm{A})$ or anti$\mathrm{CD} 3 / \mathrm{CD} 28(\mathbf{B})$ in the absence or presence of $\mathrm{N}-\mathrm{Lac}$ or dexamethasone (Dexa; $10 \mu \mathrm{mol} / \mathrm{L}$ ). Lymphoproliferation was assessed by ${ }^{3} \mathrm{H}$-thymidine uptake. C: En face adhesion assay was performed using aorta fragments and splenocytes from wildtype or Gal-3 knockout mice, in the absence or presence of $\mathrm{N}$-Lac. Data show the frequency of adherent cells 30 minutes after incubation. D: Mouse splenocytes were submitted to starvation and placed in the upper compartment of a transwell system in the presence or absence of 10 $\mu \mathrm{mol} / \mathrm{L} \mathrm{N}$-Lac. Medium with or without fetal bovine serum (FBS) was placed in the lower chamber as a chemoattractant. Cell concentration in the lower chamber after overnight incubation. Data are expressed as means \pm SEM (A-D). ${ }^{*} P<0.05,{ }^{* *} P<0.01$, and ${ }^{* * *} P<0.001$. CPM, counts per minute. of heart inflammation and fibrosis in $T$. cruzi-infected mice. This was achieved by the following: i) immunostaining in chronic Chagas disease human and mouse heart samples showing the presence of Gal- $3^{+}$cells, including macrophages, T cells, fibroblasts, and fibrocytes; ii) blockade of Gal-3 expression in cardiac fibroblasts, showing its role on proliferation and collagen production; and iii) pharmacological blockade in vivo in the experimental model, showing significant reduction of inflammation, fibrosis, and production of key inflammatory mediators in the heart.

Previous studies have highlighted a role for Gal-3 in the cardiac remodeling process in different experimental settings, including experimental models of hypertrophic cardiomyopathy and myocardial infarction. ${ }^{12-14}$ These reports have focused on Gal-3 effects in cardiac fibroblasts, contributing to cell survival, proliferation, and extracellular matrix synthesis. In CCC, however, a massive infiltration of immune cells is observed in the heart, which leads to persistent immune-mediated myocyte damage, ultimately triggering a progressive fibrogenic response. ${ }^{6-9}$

In the present study, we demonstrated the dynamic expression of Gal-3 in different periods during T. cruzi experimental infection and correlated with the findings of human heart analysis, in sections obtained from hearts of subjects with end-stage heart failure due to CCC. Gal-3 expression was observed in a similar pattern in human and mouse heart samples, mainly in areas of inflammatory infiltrates. Gal-3 has been previously described in immune cells and to participate in different aspects of innate and adaptive immune responses. ${ }^{21-26}$ In the experimental model, we showed expression of Gal-3 in macrophages and
T cells, two main cell types present in the inflammatory foci in Chagas disease hearts. Moreover, we demonstrated that inflammatory stimuli increase the expression of Gal-3 in macrophages in vitro. Because IFN- $\gamma$ and tumor necrosis factor- $\alpha$ are produced in mouse hearts chronically infected with $T$. cruzi, their action may account for the increased Gal-3 expression in macrophages. The described roles for Gal-3 in T-cell biology include the promotion of cell survival, proliferation, T-cell receptor signaling, and migration. $^{27}$ In our study, we observed reduction of cell adhesion to endothelium and migration, but not of lymphocyte proliferation, by the Gal-3 inhibitor N-Lac, suggesting that the reduction of inflammation in the hearts of infected mice after N-Lac treatment is mainly because of reduction of cell migration.

In our study, we found that $T$. cruzi infection also increased the expression of Gal-3 in cardiac fibroblasts and, even more intensely, in a population of bone marrowderived fibrocytes. Although cardiac fibroblasts have been classically described as the most important cell type involved in cardiac fibrosis, different studies have shown that bone marrow-derived fibrocytes play relevant roles in fibrogenesis and remodeling. ${ }^{28-30}$ Our data provided from in vitro assays in cardiac fibroblasts demonstrated the role of exogenous and endogenous Gal-3 in cell survival, proliferation, and type I collagen synthesis, which is supported by the current literature. ${ }^{12-14}$ The fact that extracellular Gal-3 increased cell proliferation only in high concentration and the marked reduction of proliferation in Gal-3 knockdown cells indicate that intracellular Gal-3 has a critical role in cell proliferation regulation. Interestingly, Gal-3 has been 
previously shown to enhance cyclin D1 promoter activity, ${ }^{31}$ correlating with the cell cycle arrest and decreased expression of cyclin D1 gene in Gal-3 knockdown fibroblasts found in our study. The fact that caveolin-1 gene expression was not increased in Gal-3 knockdown cells reinforces a direct action of Gal-3 in the regulation of cyclin D1 gene transcription.

We have previously shown that Lgals 3 gene expression is up-regulated in the hearts of mice during chronic T. cruzi infection. ${ }^{9,16}$ The correlation between intensity of myocarditis and presence of collagen type I, Gal-3, and $\alpha$-smooth muscle actin-positive cells was also seen in a mouse model of $T$. cruzi infection. ${ }^{32}$ Gal-3 was implicated in the process of $T$. cruzi invasion. ${ }^{33}$ Altogether, these data suggest that Gal-3 is involved in different aspects of the pathogenesis of CCC, from T. cruzi infection to immune response, inflammation, and tissue repair. Interestingly, a reduction of Gal-3 expression in the heart was observed accompanying decreased fibrosis and myocarditis after granulocyte colonystimulating factor treatment or cell therapy in chronically infected mice. ${ }^{16,34}$ In the present study, we showed that the Gal-3 pharmacological blockade with N-Lac significantly modulated the immune response in the hearts from CCC mice, reducing migration of immune cells to the myocardium and decreasing the expression of inflammatory type 1 helper T-cell cytokines and markers of type 2 helper T-cell and $\mathrm{T}$ regulatory cell lymphocyte subtypes, to the level of naïve control mice. Notably, the anti-inflammatory cytokine IL-10 was increased when compared to naïve mice. This finding, together with the observed reduced levels of IFNG and TNFA gene expression, demonstrates a potent antiinflammatory effect of N-Lac. Moreover, N-Lac treatment was associated with a significant reduction of myocardial fibrosis, which is in accordance with a previous report in a different experimental model. ${ }^{14}$ Despite the reduction of inflammation and fibrosis, our results did not correlate with any improvement in functional parameters after N-Lac treatment. This finding does not exclude the possibility of long-term beneficial effects of Gal-3 blockade, nor that $\mathrm{N}$-Lac treatment, at an earlier stage of the infection, which may prevent the deterioration of cardiac function.

The strong binding affinity between galectin- 3 and $\mathrm{N}$-acetyl-D-lactosamine has been previously reported. ${ }^{35}$ Moreover, in previous studies, similar dose and administration regimen of $\mathrm{N}$-acetyl-D-lactosamine were used to block galectin-3 in mouse models of viral myocarditis ${ }^{36}$ and hypertensive cardiac remodeling. ${ }^{14}$ The reduction of inflammation and fibrosis observed after N-Lac treatment were not observed in mice treated with sucrose in the same dose and regimen. Moreover, pharmacological (by N-Lac) and genetic (gene knockout) blockade of Gal-3 had similar effects in cell adhesion to endothelium, and indicate that $\mathrm{N}$-Lac does not interfere with selectin binding.

In a translational perspective, Gal-3 could be used in the clinical setting as either a novel biomarker or a therapeutic target. Although the identification of novel noninvasive biomarkers that adequately predict cardiac fibrosis would be highly desired, in a recent report we showed that plasma Gal-3 levels do not correlate with the intensity of fibrosis, as measured by magnetic resonance imaging, in a recently published transversal study in subjects with CCC. ${ }^{37}$ Nonetheless, these data do not exclude the possibility of Gal-3 being useful as a biomarker for prognosis determination, which is currently under investigation in chronic heart failure caused by other etiologies. ${ }^{38}$ Thus, the conduction of a longitudinal study in Chagas disease subjects would be required to validate the use of plasma Gal-3 as prognosis biomarker.

In conclusion, herein, we demonstrated that Gal-3 plays an important role in the pathogenesis of experimental chronic Chagas disease, acting in different cell compartments and promoting cardiac inflammation and fibrosis. The finding of Gal-3 expression in human heart samples, in a similar pattern as observed in the mouse model, reinforces its potential as a novel target for drug and therapy development for CCC.

\section{Acknowledgments}

We thank Pamela Daltro for technical assistance in the cardiac functional analysis, Didier Trono for pLVTHM lentiviral vector, Dr. Luiz R. Goulart for providing galectin-3 knockout mice, and Drs. Igor Correia de Almeida and Washington Luis Conrado dos Santos for helpful discussions.

\section{Supplemental Data}

Supplemental material for this article can be found at http://dx.doi.org/10.1016/j.ajpath.2017.01.016.

\section{References}

1. Andrade DV, Gollob KJ, Dutra WO: Acute Chagas disease: new global challenges for an old neglected disease. PLoS Negl Trop Dis 2014, 8:e3010

2. WHO: Chagas disease in Latin America: an epidemiological update based on 2010 estimates. Wkly Epidemiol Rec 2015, 90:33-44

3. Bern C, Montgomery SP, Herwaldt BL, Rassi A Jr, Marin-Neto JA, Dantas RO, Maguire JH, Acquatella H, Morillo C, Kirchhoff LV, Gilman RH, Reyes PA, Salvatella R, Moore AC: Evaluation and treatment of Chagas disease in the United States: a systematic review. JAMA 2007, 298:2171-2181

4. Morillo CA, Marin-Neto JA, Avezum A; BENEFIT Investigators: Randomized trial of Benznidazole for chronic Chagas' cardiomyopathy. N Engl J Med 2015, 373:1295-1306

5. Burgos JM, Diez M, Vigliano C, Bisio M, Risso M, Duffy T, Cura C, Brusses B, Favaloro L, Leguizamon MS, Lucero RH, Laguens R, Levin MJ, Favaloro R, Schijman AG: Molecular identification of Trypanosoma cruzi discrete typing units in end-stage chronic Chagas heart disease and reactivation after heart transplantation. Clin Infect Dis 2010, 51:485-495

6. Soares MB, Pontes-De-Carvalho L, Ribeiro-Dos-Santos R: The pathogenesis of Chagas' disease: when autoimmune and parasite-specific immune responses meet. An Acad Bras Cienc 2001, 73:547-559 
7. Bonney KM, Engman DM: Autoimmune pathogenesis of Chagas heart disease: looking back, looking ahead. Am J Pathol 2015, 185:1537-1547

8. Gomes JA, Bahia-Oliveira LM, Rocha MO, Martins-Filho OA, Gazzinelli G, Correa-Oliveira R: Evidence that development of severe cardiomyopathy in human Chagas' disease is due to a Th1-specific immune response. Infect Immun 2003, 71:1185-1193

9. Soares MB, de Lima RS, Rocha LL, Vasconcelos JF, Rogatto SR, dos Santos RR, Iacobas S, Goldenberg RC, Iacobas DA, Tanowitz HB, de Carvalho AC, Spray DC: Gene expression changes associated with myocarditis and fibrosis in hearts of mice with chronic chagasic cardiomyopathy. J Infect Dis 2010, 202:416-426

10. de Boer RA, Voors AA, Muntendam P, van Gilst WH, van Veldhuisen DJ: Galectin-3: a novel mediator of heart failure development and progression. Eur J Heart Fail 2009, 11:811-817

11. Krześlak A, Lipińska A: Galectin-3 as a multifunctional protein. Cell Mol Biol Lett 2004, 9:305-328

12. Sharma U, Pokharel S, van Brakel TJ, van Berlo JH, Cleutjens JP, Schroen B, André S, Crijns HJ, Gabius HJ, Maessen J, Pinto YM: Galectin-3 marks activated macrophages in failure-prone hypertrophied hearts and contributes to cardiac dysfunction. Circulation 2004, 110:3121-3128

13. González GE, Cassaglia P, Noli Truant S, Fernández MM, Wilensky L, Volberg V, Malchiodi EL, Morales C, Gelpi RJ: Galectin-3 is essential for early wound healing and ventricular remodeling after myocardial infarction in mice. Int J Cardiol 2014, 176:1423-1425

14. Yu L, Ruifrok WP, Meissner M, Bos EM, van Goor H, Sanjabi B, van der Harst P, Pitt B, Goldstein IJ, Koerts JA, van Veldhuisen DJ, Bank RA, van Gilst WH, Silljé HH, de Boer RA: Genetic and pharmacological inhibition of galectin-3 prevents cardiac remodeling by interfering with myocardial fibrogenesis. Circ Heart Fail 2013, 6:107-117

15. Chen A, Hou W, Zhang Y, Chen Y, He B: Prognostic value of serum galectin-3 in patients with heart failure: a meta-analysis. Int J Cardiol 2015, 182:168-170

16. Soares MBP, Lima RS, Souza BSF, Vasconcelos JF, Rocha LL, dosSantos RR, Iacobas S, Goldenberg RC, Lisanti MP, Iacobas DA, Tanowitz HB, Spray DC, Campos-de-Carvalho AC: Reversion of gene expression alterations in hearts of mice with chronic chagasic cardiomyopathy after transplantation of bone marrow cells. Cell Cycle 2011, 10:1448-1455

17. Committee for the Update of the Guide for the Care and Use of Laboratory Animals; National Research Council: Guide for the Care and Use of Laboratory Animals: Eighth Edition. Washington, DC, National Academies Press, 2011

18. Wiznerowicz M, Trono D: Conditional suppression of cellular genes: lentivirus vector-mediated drug-inducible RNA interference. J Virol 2003, 77:8957-8961

19. Karasuyama H, Melchers F: Establishment of mouse cell lines which constitutively secrete large quantities of interleukins 2, 3, 4, or 5 using modified cDNA expression vectors. Eur J Immunol 1988, 18:97-104

20. Kleshchenko YY, Moody TN, Furtak VA, Ochieng J, Lima MF, Villalta F: Human galectin-3 promotes Trypanosoma cruzi adhesion to human coronary artery smooth muscle cells. Infect Immun 2004, 72 : 6717-6721

21. Henderson NC, Sethi T: The regulation of inflammation by galectin-3. Immunol Rev 2009, 230:160-171

22. Hsu DK, Yang RY, Pan Z, Yu L, Salomon DR, Fung-Leung WP, Liu FT: Targeted disruption of the galectin-3 gene results in attenuated peritoneal inflammatory responses. Am J Pathol 2000, 156:1073-1083
23. Jeon SB, Yoon HJ, Chang CY, Koh HS, Jeon SH, Park EJ: Galectin-3 exerts cytokine-like regulatory actions through the JAK-STAT pathway. J Immunol 2010, 185:7037-7046

24. MacKinnon AC, Farnworth SL, Hodkinson PS, Henderson NC, Atkinson KM, Leffler H, Nilsson UJ, Haslett C, Forbes SJ, Sethi T: Regulation of alternative macrophage activation by galectin-3. J Immunol 2008, 180:2650-2658

25. Yang RY, Hsu DK, Liu FT: Expression of galectin-3 modulates t-cell growth and apoptosis. Proc Natl Acad Sci U S A 1996, 93:6737-6742

26. Chen SS, Sun LW, Brickner H, Sun PQ: Downregulating galectin-3 inhibits proinflammatory cytokine production by human monocytederived dendritic cells via RNA interference. Cell Immunol 2015 , 294:44-53

27. Tribulatti MV, Figini MG, Carabelli J, Cattaneo V, Campetella O: Redundant and antagonistic functions of galectin-1, -3 , and -8 in the elicitation of t cell responses. J Immunol 2012, 188:2991-2999

28. Chu PY, Mariani J, Finch S, McMullen JR, Sadoshima J, Marshall T, Kaye DM: Bone marrow-derived cells contribute to fibrosis in the chronically failing heart. Am J Pathol 2010, 176:1735-1742

29. van Amerongen MJ, Bou-Gharios G, Popa E, van Ark J, Petersen AH, van Dam GM, van Luyn MJ, Harmsen MC: Bone marrow-derived myofibroblasts contribute functionally to scar formation after myocardial infarction. J Pathol 2008, 214:377-386

30. Haudek SB, Cheng J, Du J, Wang Y, Hermosillo-Rodriguez J, Trial J, Taffet GE, Entman ML: Monocytic fibroblast precursors mediate fibrosis in angiotensin-II-induced cardiac hypertrophy. J Mol Cell Cardiol 2010, 49:499-507

31. Lin HM, Pestell RG, Raz A, Kim HR: Galectin-3 enhances cyclin d(1) promoter activity through $\mathrm{sp} 1$ and a camp-responsive element in human breast epithelial cells. Oncogene 2002, 21:8001-8010

32. Ferrer MF, Pascuale CA, Gomez RM, Leguizamón MS: DTU I isolates of Trypanosoma cruzi induce upregulation of galectin-3 in murine myocarditis and fibrosis. Parasitology 2014, 141:849-858

33. Machado FC, Cruz L, da Silva AA, Cruz MC, Mortara RA, RoqueBarreira MC, da Silva CV: Recruitment of galectin-3 during cell invasion and intracellular trafficking of Trypanosoma cruzi extracellular amastigotes. Glycobiology 2014, 24:179-184

34. Vasconcelos JF, Souza BS, Lins TF, Garcia LM, Kaneto CM, Sampaio GP, de Alcântara AC, Meira CS, Macambira SG, Ribeiro-dos-Santos R, Soares MB: Administration of granulocyte colony-stimulating factor induces immunomodulation, recruitment of $\mathrm{T}$ regulatory cells, reduction of myocarditis and decrease of parasite load in a mouse model of chronic Chagas disease cardiomyopathy. FASEB J 2013, 27:4691-4702

35. von Mach T, Carlsson MC, Straube T, Nilsson U, Leffler H, Jacob R: Ligand binding and complex formation of galectin-3 is modulated by pH variations. Biochem J 2014, 457:107-115

36. Jaquenod De Giusti C, Ure AE, Rivadeneyra L, Schattner M, Gomez RM: Macrophages and galectin 3 play critical roles in CVB3induced murine acute myocarditis and chronic fibrosis. J Mol Cell Cardiol 2015, 85:58-70

37. Noya-Rabelo MM, Larocca TF, Macêdo CT, Torreão JA, Souza BS, Vasconcelos JF, Souza LE, Silva AM, Ribeiro Dos Santos R, Correia LC, Soares MB: Evaluation of Galectin-3 as a novel biomarker for Chagas cardiomyopathy. Cardiology 2017, 136:33-39

38. Peacock WF: How galectin-3 changes acute heart failure decision making in the emergency department. Clin Chem Lab Med 2014, 52: 1409-1412 